\title{
Simulations of the performance of the HTRS on IXO
}

\author{
Christian Schmid $^{* a}$, Jörn Wilms ${ }^{a}$, Tim Oosterbroek $^{b}$, Michael Martin $^{c}$, \\ Eckhard Kendziorra $^{c}$, Benjamin Mück ${ }^{c}$, Didier Barret ${ }^{d}$, Damien Rambaud ${ }^{d}$, \\ Peter Lechner ${ }^{e}$ \\ ${ }^{a}$ Dr. Karl Remeis-Observatory \& ECAP, University Erlangen-Nuremberg, Germany \\ ${ }^{b}$ Advanced Studies and Technology Preparation Division, ESTEC, ESA \\ ${ }^{c}$ Institute for Astronomy and Astrophysics Tübingen, Germany \\ ${ }^{d}$ Institut de Recherche en Astrophysique et Planétologie, France \\ ${ }^{e}$ PNSensor GmbH, Germany \\ E-mail: christian.schmidesternwarte.uni-erlangen.de
}

\begin{abstract}
We present the results of our analysis of the performance of the High Time Resolution Spectrometer (HTRS) studied with a Monte-Carlo simulation of the photon imaging and detection processes. The simulation of the photon imaging is based on a model of the X-ray optics with the particular effective area distribution of the International X-ray Observatory (IXO) Silicon Pore Optics (SPO) design. The detector was simulated with analog and with digital readout electronics.

We have analysed the photon distribution on the HTRS detector at its particular out-of-focus position taking into account instrument misalignment effects within the margins imposed by the instrument requirements. We have studied different pixel geometries and analysed the bright source performance with respect to pile-up.
\end{abstract}

Fast $X$-ray timing and spectroscopy at extreme count rates

February 7-11, 2011

Champéry, Switzerland

\footnotetext{
* Speaker.
} 


\section{Introduction}

The HTRS [1] on IXO consists of an array of 31 Silicon Drift Detectors (SDD) [2]. In order to prevent split events between neighboring pixels the detector has an absorbing mask with a spoke width of $200 \mu \mathrm{m}$ on top of the edges of the SDD pixels. The HTRS is in particular designed for observations of bright galactic sources with high spectral and temporal resolution. Due to the large effective area of the IXO telescope it must be able to cope with photon rates up to the order of a few 100000 photons per second. Despite of the fast operation of the SDD's the incident photon beam has to be distributed among several pixels in order to prevent pile-up at these high rates. Therefore the detector is mounted at a particular distance behind the focal plane, such that the photons are not focussed to a small spot as for an imaging device, but are spread over a wider range covered by several pixels. The resulting photon distribution on the detector can be investigated with a MonteCarlo simulation, which also allows to study the impact of misalignment and photon pile-up on the instrument performance.

\section{Simulation Setup}

Based on a simplified model of the geometrical setup of the IXO mirror system, which is presented in Fig. 1(a), we have implemented the photon imaging process in a Monte-Carlo simulation in order to determine the distribution of X-ray photons on the detector plane of the HTRS. For the mirrors a system of multiple shells with azimuthal symmetry is assumed. The radial effective area profile is energy-dependent, as shown in Fig. 1(b).

The simulations have been performed for samples of monochromatic X-ray photons on a $1 \mathrm{keV}$ grid in the range of $1 \mathrm{keV}$ to $10 \mathrm{keV}$ with a photon surface density of $6 \cdot 10^{8}$ photons per $\mathrm{m}^{2}$ effective area. The photons are distributed among the mirror shells according to the corresponding relative effective area. Assuming perfect mirrors an incident photon after the reflection on a particular mirror shell at the position $\left(x_{\mathrm{m}}, y_{\mathrm{m}}\right)$ is moving on a straight line through the focal spot. Since the HTRS is mounted at a distance $d=11.3 \mathrm{~cm}$ behind the focal plane, the intersection of the photon path with the detector plane at $\left(x_{\mathrm{d}}, y_{\mathrm{d}}\right)$ is not located on the telescope axis, but is slightly off-axis depending on the respective mirror shell. The relation between the impact positions on the mirror and on the detector can be determined as:

$$
\left(\begin{array}{l}
x_{\mathrm{d}} \\
y_{\mathrm{d}}
\end{array}\right)=\frac{d}{f} \cdot\left(\begin{array}{l}
x_{\mathrm{m}} \\
y_{\mathrm{m}}
\end{array}\right)
$$

Without blurring effects the image of a sufficiently large sample of photons on the detector would resemble the effective area distribution, as shown in Fig. 2(a). In reality azimuthal effects due to the modular assembly of the SPO [3] as well as blurring due to imperfectly manufactured mirrors play a considerable role. In the simulation the latter are implemented by randomizing the photon path from the mirrors to the detector with a 2-dimensional Gaussian distribution with a Half Energy Width (HEW) of 5 arcsec. Therefore the photon spot does not exhibit the shell structure shown in Fig. 2(a) but looks like in Fig. 2(b).

According to Eq. (2.1) and the effective area distribution in Fig. 1(b) the photon spot has its largest extent for $1 \mathrm{keV}$ photons and gets narrower for higher photon energies, since the effective 


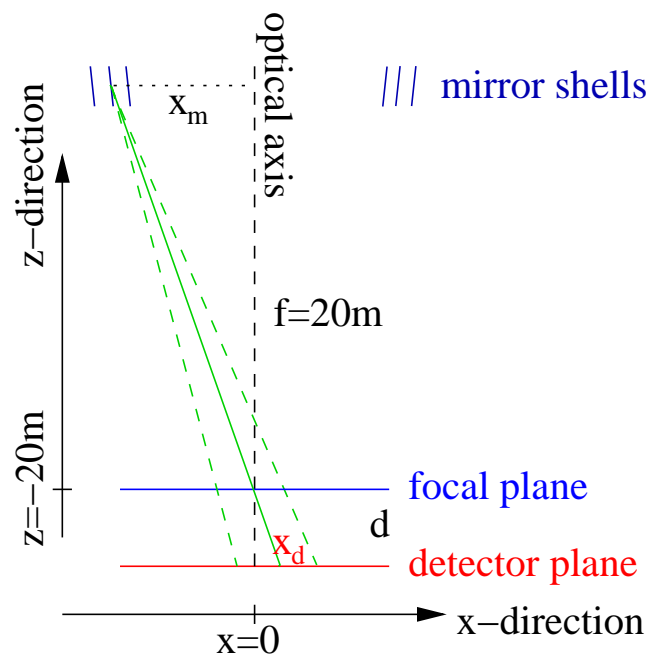

(a) Sketch of the photon path (green) from the reflecting mirror shell (dark blue) to the detector plane (red). In order to account for imperfect X-ray optics the intersection of the photon path with the focal plane (light blue) is randomized with a 2-dimensional Gaussian distribution with a HEW of 5 arcsec (indicated by the cone).

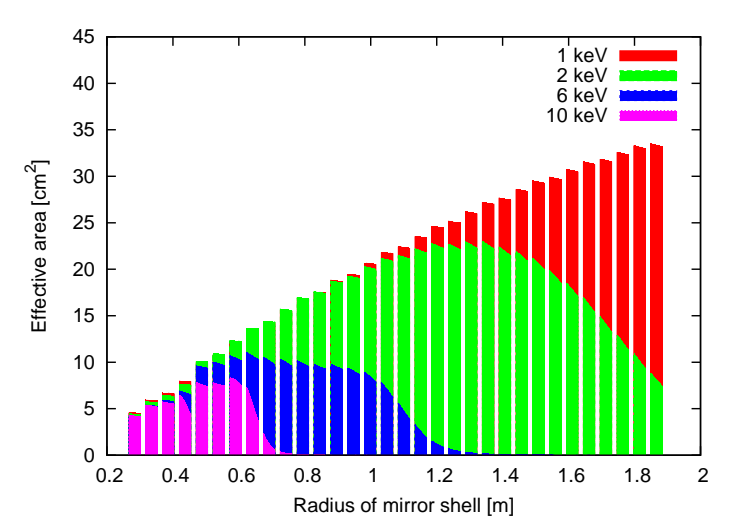

(b) Effective area distribution among the individual mirror shells of the SPO opctics for different photon energies of 1 , 2,6 , and $10 \mathrm{keV}$. The data was obtained for the setup with $3 \mathrm{~m}^{2}$ effective area at $1 \mathrm{keV}$.

Figure 1: Model of the IXO X-ray Optics used in the Monte-Carlo simulation.

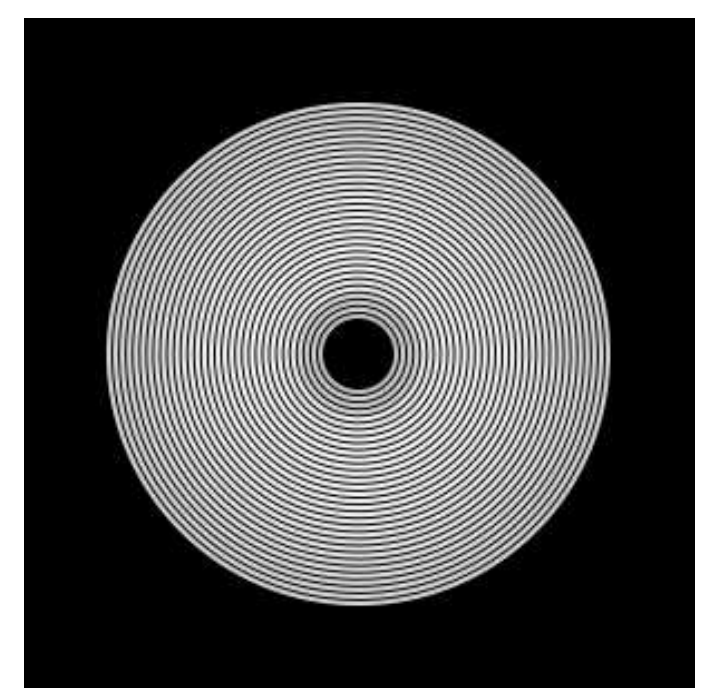

(a) Assuming perfectly radially symmetric mirrors without blurring the image on the detector reflects the shell structure.

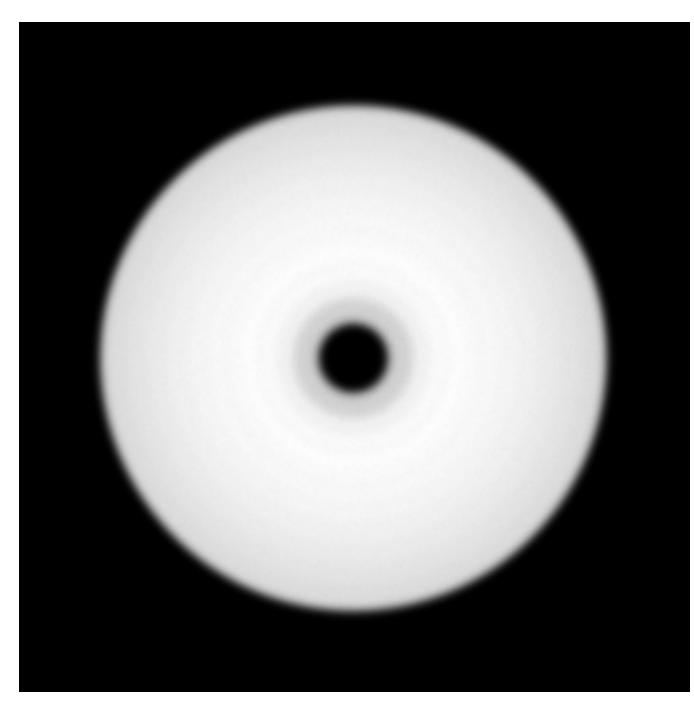

(b) In reality the photon spot is smeared due to blurring effects caused by imperfect mirrors.

Figure 2: The photon spot on the detector resembles the effective area distribution of the applied model for the SPO design. The presented data are obtained from a simulation with monochromatic $1 \mathrm{keV}$ photons. 
area of the outer mirror shells decreases. Therefore the misalignment analysis presented in this article has been performed for the most restrictive case of monochromatic $1 \mathrm{keV}$ photons.

\section{Photon Distribution \& Misalignment}

The optical setup presented in the previous section has been used to simulate the photon distribution on the detector plane of the HTRS. Based on the simulated data the pixel geometry consisting of 31 pixels with identical area, which is presented in Fig. 3, has been selected for the HTRS. This configuration is a trade-off between calibration issues and a preferably homogeneous distribution of the incident photons among the individual SDD's.

Based on this pixel geometry we have investigated the impact of detector misalignment. In the simulation the detector can be easily shifted and tilted with respect to its nominal position in order to analyse the shape and extent of the resulting photon spot. In the case of nominal alignment the center of the detector is located on the telescope axis at a distance of $11.3 \mathrm{~cm}$ behind the focal plane. The detector plane is perpendicular to the telescope axis. According to the HTRS / Platform Interface Requirement Document [4] the following alignment margins with respect to the nominal position have to be taken into account:

- horizontal position with respect to the telescope axis: $\pm 1 \mathrm{~mm}$

- vertical out-of-focus position $\pm 1 \mathrm{~mm}$

- tilt of the detector plane: $\pm 2^{\circ}$

A worst case scenario has been constructed by considering these margins. The right-hand plot in Fig. 3 displays the corresponding calculated photon spot. The selected dimensions of the HTRS are appropriate to cover the entire photon spot in the case of nominal as well as worst case alignment, as pointed out by the images in Fig. 3 .

\section{Jitter}

In addition to the static misalignment, presented in the previous section, the HTRS might be exposed to vibrations caused by other instruments on the Movable Instrument Platform (MIP) of IXO. In that case the photon spot shown in Fig. 3 is oscillating relative to the pixel array, which might be reflected by the count rates in individual pixels or by the total count rate of the instrument, because during the oscillation different parts of the photon spot are absorbed by the detector mask.

The simulated count rates for a horizontal oscillation of the detector position with an amplitude of $100 \mu \mathrm{m}$ and a frequency of $100 \mathrm{~Hz}$ during an observation with monochromatic $1 \mathrm{keV}$ photons are displayed in Fig. 4(a). The count rate variation is significant for the outer ring of pixels, because the edge of the photon spot lies in this region (cp. Fig. 3). Therefore small horizontal shifts have a strong impact on the relative change of the count rate in these pixels. However, the effect of the oscillation on the total count rate of the instrument is negligible.

One has to emphasize that the applied radially symmetric model of the mirror system is a rough simplification. In reality vibrations might have a stronger impact due to the particular modular structure of the SPO. Nevertheless the presented approach provides a first estimate of the resulting phenomena, especially for the total instrument count rate. 

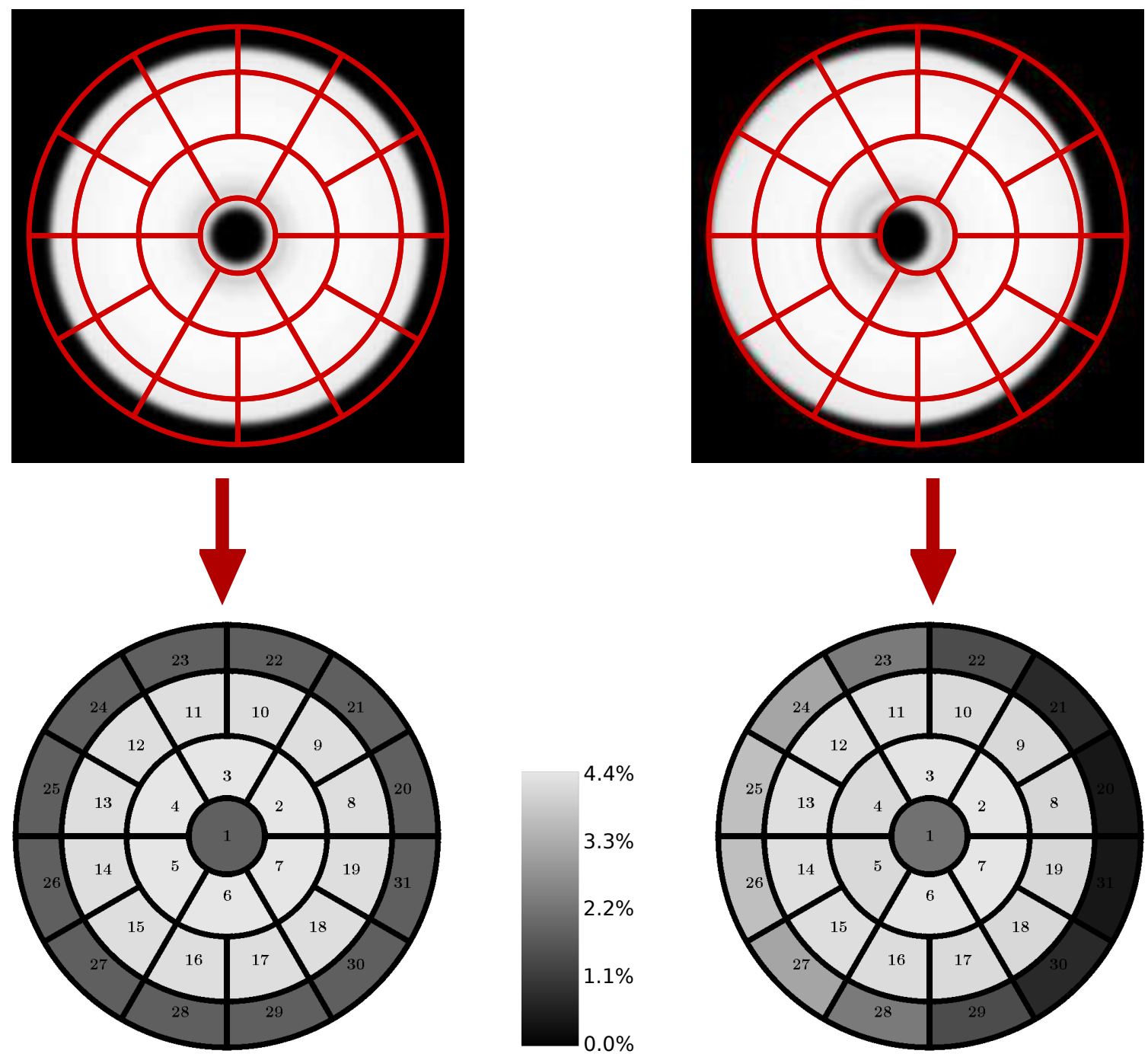

Figure 3: Distribution of $1 \mathrm{keV}$ photons on the HTRS for nominal (left-hand) and worst case (right-hand) alignment. The brightness scale of the images reflects the fraction of photons, whereas the numbers are used to identify the pixels. The radii in the presented geometry $\left(r_{1}=2.16 \mathrm{~mm}, r_{2}=5.70 \mathrm{~mm}, r_{3}=9.39 \mathrm{~mm}\right.$, and $r_{4}=12.0 \mathrm{~mm}$ ) have been selected as a trade-off between calibration issues and a preferably homogeneous distribution of photons.

\section{Pile-up}

For the readout electronics of the HTRS an analog and a digital implementation are investigated. In comparison to the analog design the digital concept has a higher power consumption but provides more flexibility, since it can be adjusted in flight, and a potentially better performance, as shown in Fig. 4(b). Both setups implement a slow and a fast filter. The slow filter is used for accurate energy determination of the detected photons, whereas the fast filter can distinguish temporally close-by photon events in order to prevent wrong detections due to pile-up. However, for observations of very bright sources there are still some pile-up events that cannot be distinguished even with the fast filter. These events are caused by several photons, but are detected as a single 


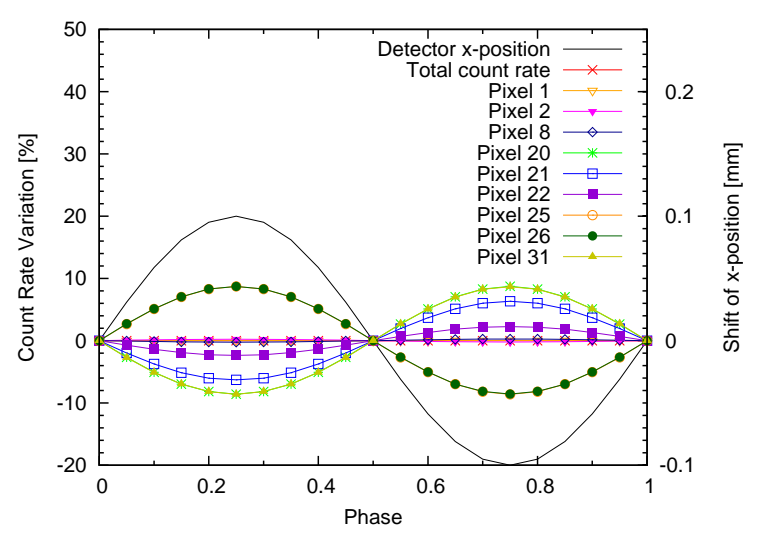

(a) Variation of the HTRS count rate due to vibrations causing an oscillation of the horizontal detector position.

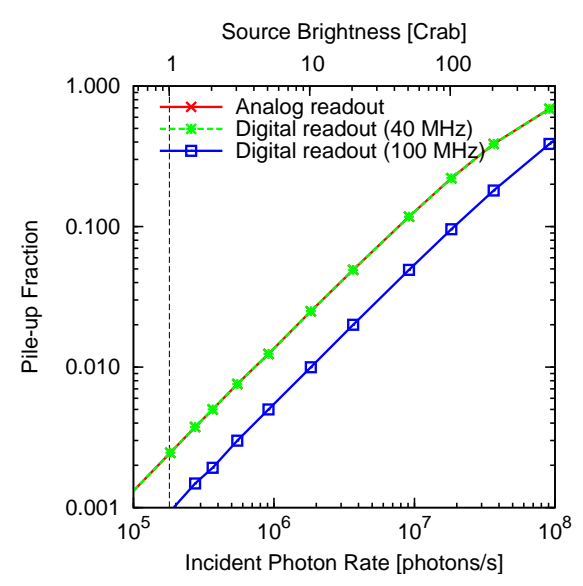

(b) Pile-up fraction among the detected events for the digital and the analog read-out electronics as a function of the source brightness.

Figure 4: Performance simulations with the presented telescope and detector models.

photon with wrong energy information.

We have simulated observations of differently bright sources with Poisson distributed photon arrival times in order to estimate the fraction of pile-up events. The resulting data are shown in Fig. 4(b) and prove that both setups with the analog and with the digital shaper fulfill the requirement of less than $2 \%$ pile-up at $1 \mathrm{Crab}$, which is given by [5].

\section{Conclusion}

Although the approach implemented in the simulation is based on some simplifying assumptions for the optical processes, it nevertheless provides a good estimate for the overall performance of the instrument. The presented data prove that the HTRS meets very well the imposed requirements with respect to the tolerance of misalignment and the pile-up performance $[4,5]$.

\section{Acknowledgments}

This research was funded by the german BMWi under DLR grant number 50 QR 0903.

\section{References}

[1] D. Barret et al., The High Time Resolution Spectrometer (HTRS) aboard the International X-ray Observatory (IXO), Proc. of SPIE 7732 (2010) 1M-1-1M-12

[2] P. Lechner et al., The silicon drift detector for the IXO high-time resolution spectrometer, Proc. of SPIE 7742 (2010) 0W-1-0W-10

[3] M. Collon et al., Stacking of silicon pore optics for IXO, Proc. of SPIE 7437 (2009) 1A-1-1A-7

[4] D. Barret et al., HTRS / Platform Interface Requirement Document, HTRS-SP-21-009-CESR (2010)

[5] D. Barret et al., HTRS contribution to IXO Payload Definition Document (PDD), HTRS-RP-21-017-CESR (2010) 